\title{
Darstellung und Struktur von Alkali-penta-tert-butoxystannaten(IV) ${ }^{1)}$
}

\author{
Michael Veith* und Michael Reimers \\ Institut für Anorganische Chemie der Universität des Saarlandes, \\ D-6600 Saarbrücken \\ Eingegangen am 26. März 1990
}

Key Words: Alkali alkoxystannates(IV) / Mixed metal alkoxides / Tin(IV), pentavalent / Coordination polymers, helical

Preparation and Structure of Alkali Penta-tert-butoxystannates(IV) ${ }^{13}$

Potassium, rubidium, and caesium tert-butoxide react with tin tetra-tert-butoxide in toluene to form compounds $\mathrm{MSn}(\mathrm{O} t \mathrm{Bu})_{5}$ $[\mathrm{M}=\mathrm{K}(2), \mathrm{Rb}(3), \mathrm{Cs}(4)]$ in contrast to lithium and sodium tertbutoxide. The compounds $2-4$ are soluble in non-coordinating solvents and seem to be almost monomeric. 2 crystallizes as $2 \cdot 1 / 2$ toluene in the orthorhombic space group Fddd. The $\mathrm{X}$-ray structure determination shows 2 to be coordinated as a helical polymer with the tin atom in the center of a trigonal bipyramid of tert-butoxide groups interlinked by two structurally different potassium atoms.
Bereits 1930 beschrieben Meerwein und Bersin ${ }^{2)}$ u. a. Alkoholate, die neben vierwertigem Zinn noch ein weiteres Metall in der Verbindung enthielten. Diese Doppelalkoxide fanden in der Folgezeit jedoch wenig Beachtung, bis vor kurzem dieser Substanzklasse, die formal aus Metallhydroxystannaten(IV) durch Substitution der Wasserstoffatome durch Organylgruppen entsteht, wieder neue Aufmerksamkeit zuteil wurde ${ }^{3-5)}$. Hampden-Smith und Mitarbeitern war es gelungen, die Existenz und die eindimensional polymere Struktur von $\left[\mathrm{Tl}_{2} \mathrm{Sn}(\mathrm{OEt})_{6}\right]_{n}$ nachzuwei$\operatorname{sen}^{4)}$.

Wir stellen im folgenden Ergebnisse vor, die wir bei der Reaktion von Zinn-tetra-tert-butoxid mit Alkali-tert-butoxiden erhielten. Diese Untersuchungen haben wir als Ergänzung und Vergleich zu unseren Arbeiten über Alkali-tertbutoxystannate(II) durchgeführt ${ }^{6}$.

Darstellung der Penta-tert-butoxystannate des Kaliums (2), Rubidiums (3) und Caesiums (4)

Kalium-, Rubidium- und Caesium-tert-butoxide reagieren in heterogener Phase mit einer Lösung von Zinn-tetratert-butoxid $\left(\mathbf{1}^{7-9)}\right.$ in Benzol gemäß Gleichung (1), wobei das vollständige Auflösen des Bodenkörpers das Ende der Reaktion anzeigt.

$$
\begin{aligned}
& \mathrm{M}+t \mathrm{BuOH} \longrightarrow \mathrm{MO} t \mathrm{Bu}+1 / 2 \mathrm{H}_{2} \\
& \mathrm{Sn}(\mathrm{O} t \mathrm{Bu})_{4}+\mathrm{MO} t \mathrm{Bu} \longrightarrow \mathrm{MSn}(\mathrm{O} t \mathrm{Bu})_{5} \\
& 12 \text { 2: } \mathrm{M}=\mathrm{K} \\
& \text { 3: } \mathrm{M}=\mathrm{Rb} \\
& \text { 4: } \mathrm{M}=\mathrm{Cs}
\end{aligned}
$$

Weder Lithium- noch Natrium-tert-butoxid bilden unter den gleichen Bedingungen mit $\mathrm{Sn}(\mathrm{O} t \mathrm{Bu})_{4}$ Doppelalkoxide: Die Reaktionskomponenten werden unzersetzt zurückerhalten. Das Ausbleiben einer Reaktion kann außer auf den Metall-Größenunterschied auf die geringere Basizität dieser Alkoholate gegenüber den Alkoholaten der schwereren Elemente erklärt werden. Es fällt zudem auf, daß im Vergleich zur Umsetzung der Metall-tert-butoxide mit Zinn-di-tertbutoxid ${ }^{6}$ die Tendenz zur Bildung von Doppelalkoxiden bei Zinn(IV) abnimmt. Zinn(II) ist im Vergleich mit Zinn(IV) die stärkere Lewis-Säure und reagiert demnach auch bereitwillig mit Lithium- und Natrium-tert-butoxid.

Die Produkte 2-4 fallen als farblose Kristalle beim Einengen des Lösungsmittels (Toluol oder Benzol) an. Nach Elementaranalyse und Röntgenstrukturanalyse (siehe folgendes Kapitel) ist das Verhältnis Alkali-Element:Zinn = 1:1. Die Verbindungen 2-4 unterscheiden sich demnach von den Doppelalkoxiden mit Ethylgruppen am Sauerstoff, die eine 2:1-Stöchiometrie aufweisen [z. B. $\mathrm{K}_{2} \mathrm{Sn}(\mathrm{OEt})_{6}{ }^{2)}$ und $\left.\mathrm{Tl}_{2} \mathrm{Sn}(\mathrm{OEt})_{6}{ }^{4}\right]$. Offenbar führt die sterisch anspruchsvollere tert-Butylgruppe zu einer Limitierung des Platzes um das Zinnatom. Auch ein Überschuß an Alkali-tert-butoxid in der Reaktion führt zu keinen neuen Produkten.

Die 'H-NMR-Spektren der Doppelalkoxide in Lösung zeichnen sich durch ein einziges Resonanzsignal aus. Im Gegensatz zum Festkörper sind die tert-Butylgruppen - zumindest im zeitlichen Mittel - chemisch äquivalent. Die Werte der Molmassenbestimmungen an 2 und 4 liegen systematisch um $30 \%$ über denjenigen der monomeren Moleküle. Im Gegensatz zur Kristallstruktur sind die MSn$(\mathrm{O} t \mathrm{Bu})_{5}$-Einheiten in Lösung nicht zu Polymeren assoziiert, sondern es sind Gleichgewichte zwischen Monomeren und Di- oder Trimeren anzunehmen.

\section{Röntgenstrukturbestimmung an $2 \cdot 1 / 2$ Toluol}

Nur von Verbindung 2, die zusammen mit einem halben Toluolmolekül pro Formeleinheit kristallisiert, konnten zur Röntgenstrukturanalyse geeignete Einkristalle erhalten werden. Zur groben Bestimmung der Gitterkonstanten und zur Raumgruppenzuordnung wurden Filmaufnahmen herangezogen. Alle wesentlichen Daten zu dem Kristall und zu der Strukturbestimmung sind in Tab. 1 zusammengestellt ${ }^{10,11}$.

In Abb. 1 ist das Ergebnis der Röntgenstrukturbestimmung von 2 dargestellt. Über Alkoholat-Brücken sind vier- 
Tab. 1. Kenngrößen zur Kristall- und Strukturbestimmung von $\mathrm{KSn}(\mathrm{O} t \mathrm{Bu})_{5} \cdot 1 / 2 \mathrm{C}_{6} \mathrm{H}_{5} \mathrm{CH}_{3}(2 \cdot 1 / 2$ Toluol $)$

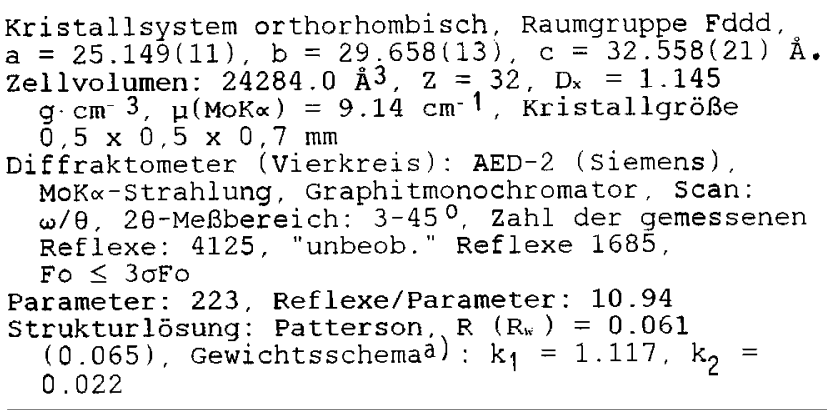

4) $w=k_{1} /\left[\sigma^{2}(F)+k_{2} F^{2}\right]$.

fach koordinierte Kaliumatome und fünffach koordinierte Zinnatome miteinander verbunden, so da $\beta$ ein eindimensionales, helixartig aufgerolltes Band $\left(\mathrm{O}_{2} \mathrm{SnO}_{2} \mathrm{~K}\right)_{n}$ entsteht. Blickt man in [ $\left[\begin{array}{lll}-1 & 0 & 0\end{array}\right]$-Richtung auf die Struktur, so erkennt man, daß die Helices versuchen, eine dichteste „Säulenpackung“ aufzubauen, wobei die Lösungsmittelmoleküle zwischen den Kettenpolymeren zu liegen kommen (Abb. 2). Die $\mathrm{C}_{6} \mathrm{H}_{5} \mathrm{CH}_{3}$-Achse weist in Richtung [ [ 1100$]$, so daß die Packung der Polymerketten in [ $\left[\begin{array}{lll}0 & 1 & 0\end{array}\right]$ - und [ $\left[\begin{array}{lll}0 & 0 & 1\end{array}\right]$-Richtung durch die anisotrope Gestalt der Toluolmoleküle unterschiedlich stark beeinflußt wird. Die Abweichung von der hexagonal dichtesten Anordnung wird dadurch verständlich.

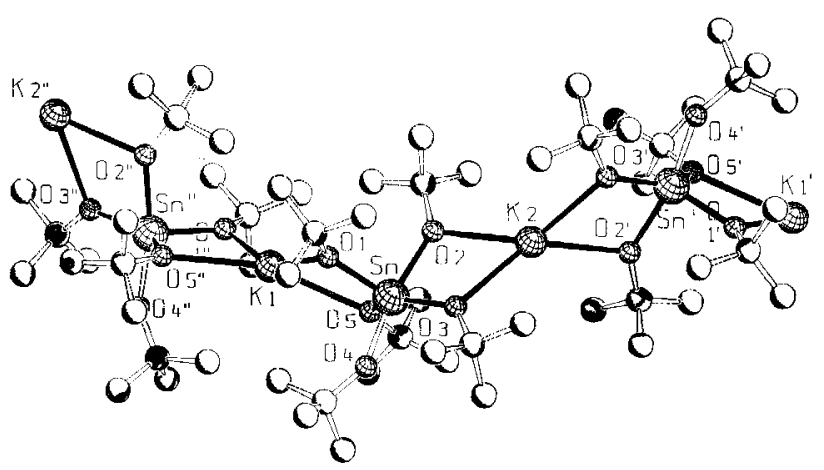

Abb. 1. Ausschnitt aus der helikalen Struktur $\left[\mathrm{KSn}(\mathrm{O} t \mathrm{Bu})_{5}\right]_{\infty}$ mit einigen Atomnumerierungen ${ }^{14}$. Die Toluolmoleküle sind weggelassen

Während das Zinnatom eine allgemeine Punktlage in der Raumgruppe einnimmt, befinden sich die beiden Kaliumatome jeweils auf zweizähligen Achsen. Diese beiden Achsen sind orthogonal zueinander angeordnet und verlaufen beide orthogonal zur 2 -Achse, um die sich die Helix windet.

In Tab. 2 sind ausgewählte Abstände und Winkel zwischen den Atomen von 2 1/2 Toluol zusammengestellt. Das fünffach von Sauerstoffatomen koordinierte Zinnatom (Abb. 1) besitzt eine Koordinationsfigur, die einer trigonalen Bipyramide recht nahe kommt (vgl. auch Daten von Tab. 2). Die Atome $O(1), O(3)$ und $O(5)$ befinden sich in äquatorialer Position, während $\mathrm{O}(2)$ und $\mathrm{O}(4)$ in den beiden axialen Stel- lungen zu liegen kommen. Von den fünf Sauerstoffatomen sind vier [nämlich $\mathrm{O}(1), \mathrm{O}(2), \mathrm{O}(3), \mathrm{O}(5)$ ] außer an tertButylgruppen auch noch an die beiden Kaliumatome gebunden, während eines $[\mathrm{O}(4)]$ nur zweifach koordiniert ist. Letzteres besitzt den größten $\mathrm{Sn}-\mathrm{O}-\mathrm{C}$-Winkel $\left[141.7(8)^{\circ}\right]$ und hat im Vergleich zum ebenfalls axial gebundenen $O(2)$ Atom einen um $0.052 \AA$ kürzeren $\mathrm{Sn}-\mathrm{O}$-Abstand. Die drei äquatorial gebundenen Sauerstoffatome unterscheiden sich in ihrem Abstand zum Zinnatom nur wenig (Tab. 2) und sind erwartungsgemäß näher am Zentralatom als das vergleichbare Sauerstoffatom $\mathrm{O}(2)$, das axial gebunden ist. Der mittlere Sn-O-Abstand beträgt für 2 $1 / 2$ Toluol 2.01(3) $\AA$ und ist damit um $0.11 \AA$ kleiner als in $\mathrm{Tl}_{2} \mathrm{Sn}(\mathrm{OtBu})_{6}{ }^{4)}$. Während diese Differenz mit den unterschiedlichen Koordinationszahlen am $\mathrm{Sn}$ in den beiden Verbindungen erklärt werden kann, überrascht dennoch der im Vergleich zu vierfach koordinierten $\operatorname{Sn}(\mathrm{IV})$-Atomen $\left(1.98 \AA^{12)}\right)$ relativ niedere Wert bei $2 \cdot 1 / 2$ Toluol.

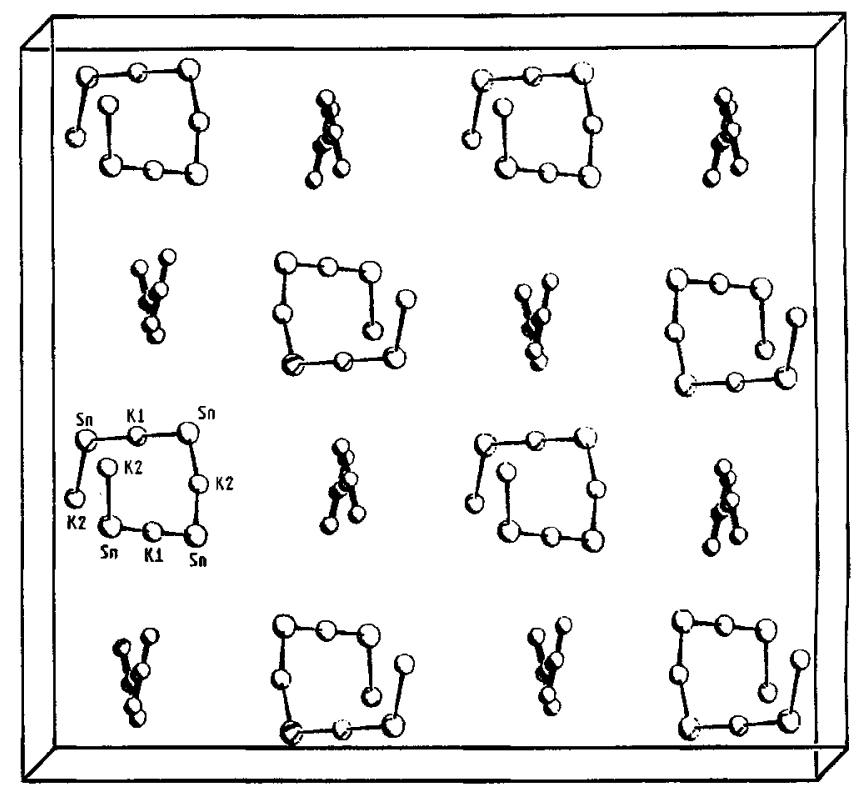

Abb. 2. Elementarzelle der Verbindung [ $\mathrm{KSn}(\mathrm{O} t \mathrm{Bu})_{5} \cdot 1 / 2$ Toluol], Blickrichtung $\left[\begin{array}{lll}-1 & 0 & 0\end{array}\right]$. Die tert-Butoxygruppen sind der Übersichtlichkeit halber weggelassen, während die Toluolmoleküle (Sechsring verläuft parallel zur Blickrichtung) dargestellt sind. Es sind sowohl links- wie rechts-drehende polymere Schrauben vorhanden. Die Sn-K-Verbindungslinien repräsentieren keine chemischen Bindungen

Von den beiden strukturell unterschiedlichen Kaliumatomen besitzt $\mathbf{K}(2)$ die kürzeren Abstände zu den Sauerstoffatomen [Mittelwerte: K(1): 2.809(6), K(2): 2.650(7) §]] und ist einer verzerrt tetraedrischen Koordinationsfigur (Bisphenoid) näher als $\mathbf{K}(1)$, das beinahe schon als planar koordiniert bezeichnet werden kann. So beträgt der Diederwinkel der $\mathrm{O}(1), \mathrm{O}(5), \mathrm{K}(1)$-Ebene zur $\mathrm{O}\left(1^{\prime \prime}\right), \mathrm{O}\left(5^{\prime \prime}\right), \mathrm{K}(1)$-Ebene $23.5^{\circ}$, während die Ebenen $\mathrm{O}(2), \mathrm{O}(3), \mathrm{K}(2)$ und $\mathrm{O}\left(2^{\prime}\right), \mathrm{O}\left(3^{\prime}\right), \mathrm{K}(2)$ einen Winkel von $30.7^{\circ}$ zueinander bilden. Die unterschiedlichen Koordinationen von $\mathrm{K}(1)$ und $\mathrm{K}(2)$ haben ihre Ursache in der gänzlich verschiedenen Chelatisierung, ausgehend von der $\mathrm{Sn}(\mathrm{O} t \mathrm{Bu})_{5}$-Einheit. Während $\mathrm{K}(1)$ von den beiden äquatorial-ständigen Sauerstoffatomen $O(1)$ und 
Tab. 2. Ausgewählte Bindungslängen $[\AA]$ und -winkel $\left[{ }^{\circ}\right]$ in 2

\section{Bindungslängen [̊]}

$\begin{array}{llll}O(1)-\mathrm{Sn} & 1.979(7) & \mathrm{O}(2)-\mathrm{Sn} & 2.057(7) \\ O(3)-\mathrm{Sn} & 2.008(7) & O(4)-\mathrm{Sn} & 2.005(8) \\ O(5)-\mathrm{Sn} & 2.002(7) & O(1)-\mathrm{K}(1) & 2.814(7) \\ O(5)-\mathrm{K}(1) & 2.803(7) & O(2)-\mathrm{K}(2) & 2.643(8) \\ O(3)-\mathrm{K}(2) & 2.656(8) & \mathrm{C}-\mathrm{O} \mathrm{a}) & 1.43(1) \\ \left.\mathrm{C}-\mathrm{Ca}^{\mathrm{a}}\right) & 1.50 & & \end{array}$

intermolekulare" nichtbindende" Wechselwirkungen

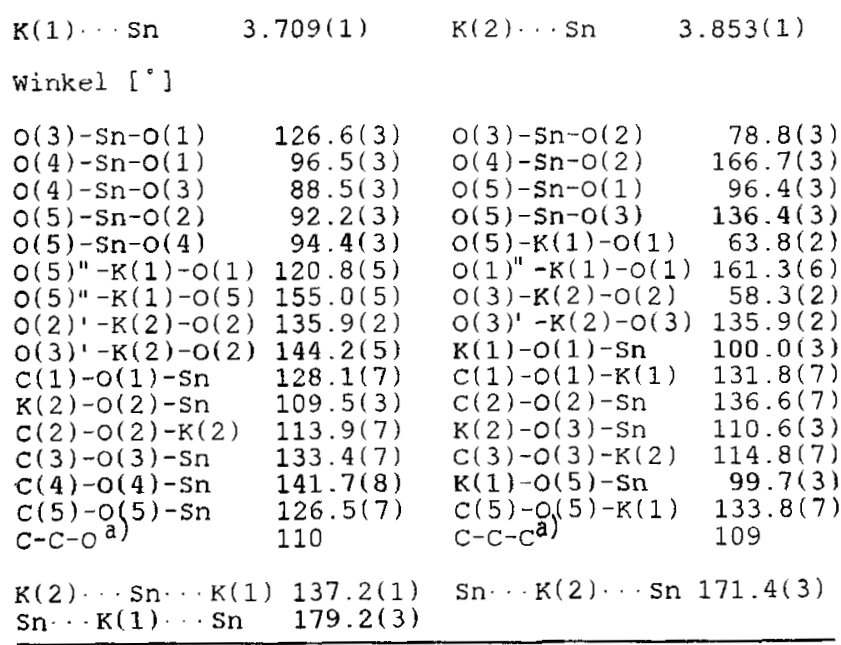

a) Mittelwert.

$\mathrm{O}(5)$ in einen viergliedrigen $\mathrm{KO}_{2} \mathrm{Sn}$-Ring eingebunden wird, entstammen die entsprechenden Sauerstoffatome $O(2)$ und $\mathrm{O}(3)$ bei $\mathrm{K}(2)$ axialer und äquatorialer Position. Der Winkel $\mathrm{O}(1)-\mathrm{Sn}-\mathrm{O}(5)$ ist $23.6^{\circ}$ kleiner als der ideale $120^{\circ}$-Winkel zwischen äquatorialen Liganden einer trigonalen Bipyramide; auf der anderen Seite weicht der $\mathrm{O}(2)-\mathrm{Sn}-\mathrm{O}(3)$ Winkel nur $11.2^{\circ}$ vom idealen Winkel zwischen äquatorialen und axialen Liganden $a b$ (Tab. 2). Die Deformation des letzteren Winkels ist also nur halb so groß. K(2) erscheint demnach effektiver von den $\mathrm{Sn}(\mathrm{O} t \mathrm{Bu})_{5}$-Einheiten gebunden $\mathrm{zu}$ sein als $K(1)$. Im Gegensatz zu den kürzeren Abständen zu den Sauerstoffatomen ist der nichtbindende $K(2) \cdots$ SnAbstand um $0.144 \AA$ länger als der $\mathrm{K}(1) \cdots$ Sn-Abstand. Hier spiegeln sich die spitzeren $\mathrm{O}-\mathrm{Sn}-\mathrm{O}$ - und $\mathrm{O}-\mathrm{K}-\mathrm{O}$ Winkel im Viereck $\mathrm{K}(1) \mathrm{O}_{2} \mathrm{Sn}$ im Vergleich zum Viereck $\mathrm{K}(2) \mathrm{O}_{2} \mathrm{Sn}$ wider (Tab. 2). Die Sauerstoffatome $\mathrm{O}(1), \mathrm{O}(2)$ und $O(5)$ sind weitgehend trigonal planar von Kalium- und Zinnatomen koordiniert (Winkelsummen: $359.9^{\circ}, 360.0^{\circ}$, $358.8^{\circ}, 360.0^{\circ}$ ).

\section{Experimenteller Teil}

Alle Reaktionen und Untersuchungen wurden unter AusschluB von Luft und Feuchtigkeit unter Schutzgas (Argon oder Stickstoff) ausgeführt. - ${ }^{1}$ H-NMR: Benzollösung, i-TMS, Gerät Bruker WP 80/Aspekt 2000 (80 MHz). - C-,H-Analysen: Mikroanalytisches Laboratorium Beller, Göttingen. - Röntgenbeugungsaufnahmen: Weissenberg-Kamera der Firma Stoe, Darmstadt. Ortskoordinaten und $B$-Werte in Tab. 3.

Kalium-penta-tert-butoxystannat(IV) (2): Zu einer Suspension von $0.19 \mathrm{~g}$ (1.70 mmol) Kalium-tert-butoxid in $10 \mathrm{ml}$ Toluol pi-
Tab. 3. Ortskoordinaten und Parameter des Debye-Waller-Faktors (Berechnung nach Lit. ${ }^{13)}$ aus $U_{i j}$ ) für die Atome der asymmetrischen Einheit von 2

\begin{tabular}{|c|c|c|c|c|}
\hline Atom & $\mathrm{x}$ & y & $z$ & $B\left[\AA^{2}\right]$ \\
\hline & $0.25169(3)$ & $0.29850(3)$ & $0.18334(2)$ & $3.99(4)$ \\
\hline & 0.1250 & & & \\
\hline (2) & $0.3750(0)$ & 0.375010 & & \\
\hline & $0.1790(3)$ & $0.3180(3)$ & $0.1984(2$ & $4.7(4)$ \\
\hline (2) & $0.2763(3)$ & $0.3622(3)$ & $0.1670(3)$ & \\
\hline 3) & $0.3176(3)$ & $0.3052(3)$ & 0.2176 & 5. \\
\hline & $0.2450(4)$ & $0.2351(3)$ & $0.2041(3)$ & $6.0(5)$ \\
\hline & (3) & & & \\
\hline ) & $0.1604(5)$ & $0.3349(5)$ & $0.2377(4)$ & 5. \\
\hline 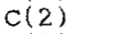 & $0.2545(6)$ & $0.3994(5)$ & $0.1456(5)$ & 6.8 \\
\hline & 0.3 & 0.27 & 0.2 & 3) \\
\hline & $0.2128(7)$ & $0.1959(5)$ & 0.200 & 7.0 \\
\hline & $0.2714(5)$ & 0.26 & & \\
\hline 1) & $0.2042(7)$ & $0.3595(6)$ & 0.2 & (4) \\
\hline 2) & 0.114 & 0.3 & & \\
\hline$C(13)$ & 0.14 & 0.2 & 0.2 & $11.0(6)$ \\
\hline & 0.2 & 0.2 & & 10. \\
\hline$c($ & 0.2 & 0.3 & 0.1226 & $6.5(3)$ \\
\hline$c($ & 0.2 & 0.4 & 0.117 & $10.4(5)$ \\
\hline$C(31)$ & 0.3 & 0.305 & 0.266 & (4) \\
\hline$C(32)$ & 0.36 & 0.2 & 0.22 & $7.9(4)$ \\
\hline & 0.3 & 0. & 0.2 & \\
\hline$C(41)$ & $0.1627(6)$ & $0.2040(5)$ & $0.1756(5)$ & $7.1(4)$ \\
\hline & 0.24 & 0.16 & 0.17 & \\
\hline$C(43)$ & $0.1987(8)$ & $0.1808(6)$ & $0.2438(6)$ & $9.3(5)$ \\
\hline$C(51)$ & $0.3229(7)$ & $0.2573(6)$ & $0.1126(6)$ & $8.9(5)$ \\
\hline & $0.2482(9)$ & 0.23 & $0.0721(7)$ & $11.1(6)$ \\
\hline C(53) & $0.279(1)$ & $0.3025(8)$ & $0.0623(7)$ & $13.2(7)$ \\
\hline & $1.159(1)$ & $0.3750(0)$ & $0.3750(0)$ & $14 .(1)$ \\
\hline$c(62)$ & $1.1720(0)$ & $0.3279(0)$ & $0.3853(0)$ & $21 .(1)$ \\
\hline & $1.2304(0)$ & $0.3279(0)$ & $0.3852(0)$ & 17.11 \\
\hline & $0(0)$ & & $0.3750(0)$ & 20. \\
\hline & $1.298(2)$ & $0.3750(0)$ & $0.3750(0)$ & $20 .(2$ \\
\hline
\end{tabular}

a) Die Positionsparameter dieser Atome wurden bei der Endverfeinerung teilweise festgehalten.

pettiert man eine Lösung von $0.70 \mathrm{~g}(1.70 \mathrm{mmol})$ Zinntetra-tertbutoxid $^{9)}$ in $10 \mathrm{ml}$ Toluol und rührt $30 \mathrm{~min}$, wobei eine klare Lösung entsteht. Das Lösungsmittel wird eingeengt, wobei $0.88 \mathrm{~g}$ $(90 \%) 2$ kristallisieren.

Farblose Kristalle, löslich in Toluol, $n$-Hexan und Diethylether, Schmp. $190^{\circ} \mathrm{C}$ (Zers.). $-{ }^{1} \mathrm{H}-\mathrm{NMR}: \delta=1.60\left[\mathrm{OC}\left(\mathrm{CH}_{3}\right)_{3}\right]$.

$$
\begin{aligned}
& \mathrm{C}_{20} \mathrm{H}_{45} \mathrm{KO}_{5} \mathrm{Sn} \mathrm{(523.3)} \\
& \text { Ber. C } 45.90 \mathrm{H} 8.67 \\
& \text { Gef. C } 44.11 \mathrm{H} 8.67 \\
& \text { Molmasse } 694 \mathrm{~g} / \mathrm{mol} \text { (kryoskop. in Benzol) }
\end{aligned}
$$

Rubidium- (3) und Caesium-penta-tert-butoxystannat (IV) (4): $\mathrm{Zu}$ einer Suspension von $2.0 \mathrm{mmol}$ Rubidium- oder Caesium-tertbutoxid in $10 \mathrm{ml}$ Toluol pipettiert man eine Lösung von $0.26 \mathrm{~g}(0.63$ mmol) Zinn-tetra-tert-butoxid ${ }^{\text {9) }}$ in $10 \mathrm{ml}$ Toluol und rührt $15 \mathrm{~min}$. Das nicht umgesetzte Rubidium- bzw. Caesium-tert-butoxid wird abfiltriert, und nach Abkondensieren des Lösungsmittels erhält man 3 bzw. 4 in quantitativer Ausbeute.

3: Farblose, verwachsene Kristalle, löslich in Benzol, Toluol, $n$-Hexan und Diethylether, Schmp. $94^{\circ} \mathrm{C} .-{ }^{1} \mathrm{H}-\mathrm{NMR}: \delta=1.61$ $\left[\mathrm{OC}\left(\mathrm{CH}_{3}\right)_{3}\right]$.

$$
\begin{array}{rr}
\mathrm{C}_{20} \mathrm{H}_{45} \mathrm{O}_{5} \mathrm{RbSn}\left(\cdot 0.5 \mathrm{C}_{7} \mathrm{H}_{8}\right)(615.8) & \text { Ber. C } 45.84 \mathrm{H} 8.02 \\
& \text { Gef. C } 47.47 \mathrm{H} 7.98
\end{array}
$$

4: Farblose, verwachsene Kristalle, löslich in Benzol, $n$-Hexan und Diethylether, Schmp. $80^{\circ} \mathrm{C} .-{ }^{1} \mathrm{H}-\mathrm{NMR}: \delta=1.61\left[\mathrm{OC}\left(\mathrm{CH}_{3}\right)_{3}\right]$.

$\mathrm{C}_{20} \mathrm{H}_{45} \mathrm{CsO}_{5} \mathrm{Sn}$ (617.2)

Ber. C 38.92 H 7.35

Gef. C 39.12 H 7.07

Molmasse $761 \mathrm{~g} / \mathrm{mol}$ (kryoskop. in Benzol) 
1) 4. Mitteilung über Alkoxystannate; 3. Mitteilung: M. Veith, P. Hobein, R. Rösler, Z. Naturforsch., Teil B, 44 (1989) 1067.

${ }^{2)}$ H. Meerwein, T. Bersin, Liebigs Ann. Chem. 476 (1929) 113 [Chem. Abstr. 24 (1930) 586].

3) D. C. Bradley, R. C. Mehrotra, D. P. Gaur, Metal Alkoxides, Academic Press, London 1978.

4) M. Hampden-Smith, O. Smith, E. Duester, Inorg. Chem. 28 (1989) 3401.

5) K. G. Caulton, Indiana University, USA, private Mitteilung.

6) M. Veith, R. Rösler, Z. Naturforsch., Teil B, 41 (1986) 1071.

7) D. C. Bradley, E. N. Caldwell, W. Wardlaw, J. Chem. Soc. 1957, 4775.

8) M. Veith, J. Pöhlmann, D. Käfer, unveröffentlichte Ergebnisse.
${ }^{9)}$ M. Reimers, Dissertation, Univ. Saarbrücken, 1990.

10) G. M. Sheldrick, SHELX-76/86, Programm for Crystal Structure Determinations, University of Cambridge, 1976.

11) Weitere Einzelheiten zu der Kristallstrukturuntersuchung können beim Fachinformationszentrum Karlsruhe, Gesellschaft für wissenschaftlich-technische Information $\mathrm{mbH}$, D-7514 Eggenstein-Leopoldshafen 2, unter Angabe der Hinterlegungsnummer CSD-54536, der Autorennamen und des Zeitschriftenzitats angefordert werden.

12) M. Veith, O. Recktenwald, Top. Curr. Chem. 104 (1982) 1.

${ }^{13)}$ W. C. Hamilton, Acta Crystallogr. 12 (1959) 609.

${ }^{14)}$ E. Keller, SCHAKAL-88, Programm zur Darstellung von Kristallstrukturen, Universität Freiburg, 1988.

$[106 / 90]$ 